\title{
SAFETY OF USE OF HIGH ALTITUDE PROTECTION SUITS FOR KINESITHERAPY - PRELIMINARY REPORT
}

\author{
Maciej ABAKUMOW' ${ }^{1}$ Krzysztof KOWALCZUK² \\ 1 Neures Poland, Warsaw, Poland \\ 2 Department of Simulator Studies and Aeromedical Training, Military Institute of Aviation Medicine, \\ Warsaw, Poland
}

Source of support: Own sources

Author's address: M. Abakumow, Neures Polska, Przasnyska 6a Street, 01-755 Warsaw, Poland, e-mail: rehabilitacja@neures.pl

Abstract: Apart from protection from very high altitude or influence of increased gravitational accelerations protective suits sometimes are used for another applications like supporting kinesitherapy. Because of some safety considerations connected with possible cardiovascular system overload and dangerous blood pressure increase we tested if these concerns are valid. Main aim ot presented research performed with participation of healthy volunteers was to confirm that use of High Altitude Protection (HAP) suit is safe in terms of increased cardiovascular.

Keywords: altitude protection suit, kinesitherapy, cardiovascular load, therapy safety 
Abakumow M. et al. - Safety of use of hight...

\section{INTRODUCTION}

High altitude protection suits (HAP suits), sometimes called also pressure suits, are devices designed and normally used for protection of aircrew in case of emergencies connected with exposition for very low atmospheric pressure (e.g. lost of cabin pressure at high altitude). The need for mechanical compression on the wearer body arises from two reasons. First, at the altitudes

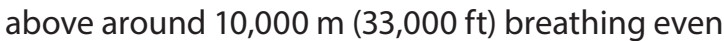
$100 \%$ oxygen under atmospheric pressure at such altitude does not meet human organism needs and oxygen must be administered under pressure greater than surrounding atmospheric pressure. Such condition, called positive pressure breathing (PPB), can lead to lung and thorax distension or even risk of lung tissue rupture hence there is need for external compression on the chest to balance intrapulmonary pressure. Secondly, at even higher altitudes - around 18-19000m (60-62000ft) there is a risk of boiling of exposed body liquids. Pressure at such altitudes is low enough (around $47 \mathrm{mmHg}$ ) to allow water boiling in temperature of human body. For protection of life at such altitudes both positive pressure breathing and external pressure on the body is critically required.

Apart from being a safety device, high altitude protection suits sometimes are used in another applications like increasing blood pressure in vertical position in person after lengthy microgravity exposure or in kinesitherapy as a supporting devices for persons with antigravitational muscles deficits [4]. Additional advantage of using body compression techniques is concurrent both muscular and proprioceptive stimulation ongoing during therapeutic session [7]. Overall view of suit is present on fig. 1, it's principle of operation is shown on fig. 2 .

Major safety concern in normobaric and nonemergency conditions is increased blood pressure and breathing difficulties caused by high mechanical pressure on the wearers body.

Use of pneumatic assembles exerting the force on body in kinesitherapy is not frequent and the bibliography associated with this subject is scarce. Main system used in this application is Russian "Atlant" system [2]. We did not find any publications concerned on safety of their use, especially in terms of possible cardiovascular overload of patients involved. Some available data $[1,6]$ suggest, that inflation below $70 \mathrm{mmHg}$ is completely safe in terms of significant blood pressure increase.

As a part of broader project in area of kinesitherapy we tried to investigate if wearing HAP suit inflated to pressures from range planned for future experiments is safe in terms of cardiovascu- lar burden put on the participants organisms. Main aim of this paper is to assess performed research results in terms of safety to the suit wearer expressed with increase of HR and RR within safe limits.

\section{MATERIAL AND METHODS}

In presented work we used WUK-90 high altitude protection suit (Air-Pol, Legionowo, Poland). It is a capstan-type suit. In this system compression over the body is done through inflating rubber tubes which are increasing pressure on the body with non distending tapes sewn into suit fabric. In addition suit is equipped with abdominal bladder exerting pressure on lower abdomen and frontal pelvic region. Precise adjustment of suit is done with use of polypropylene laces. Closing of suit is done by means of metal zippers.

Therefore increasing of air pressure in capstan system and abdominal bladder is increasing mechanical force exerted by suit on the body of person wearing it.

Before experiments we obtained written approval from Military Institute of Aviation Medicine in Warsaw Ethics Committee in accordance with the Helsinki Declaration of 1975, as revised in 2000. Each participant signed informed consent

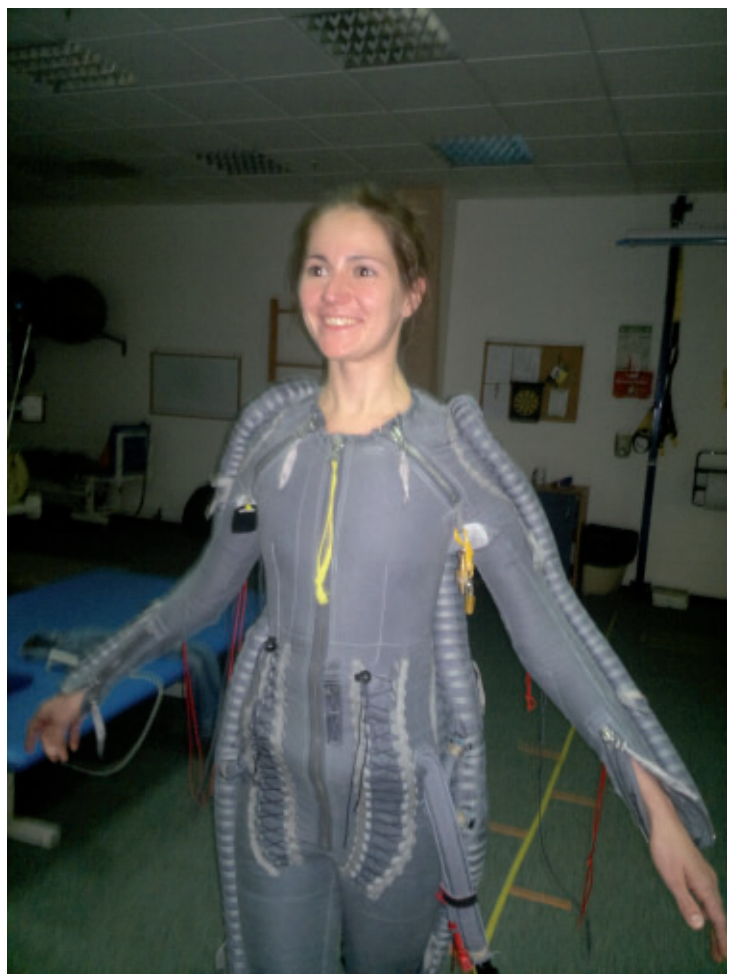

Fig. 1. WUK high altitude protection suit used in experiments (photo presented with patient's consent). 

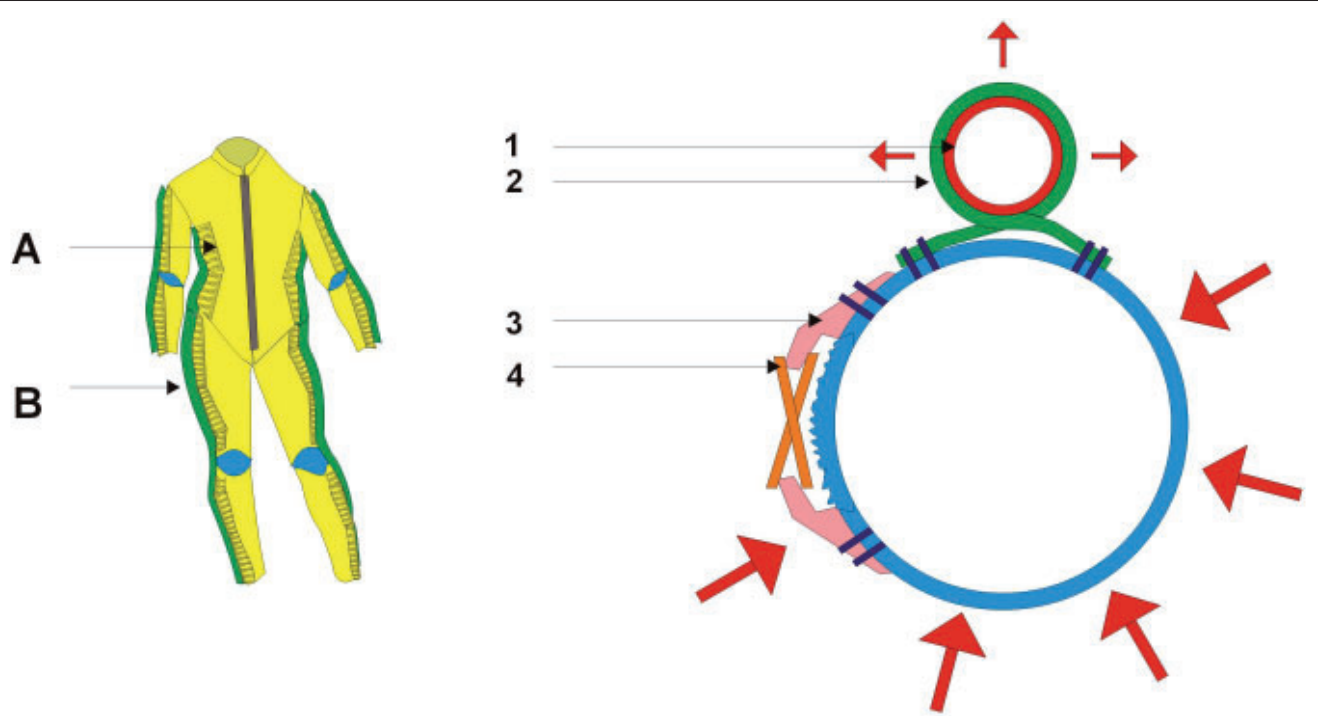

Fig. 2. Capstan type suit work principle. A-laces B capstan tube covered by fabric tapes, 1 - rubber tube 2-capstan tapes 3-laces mount 4-lace.

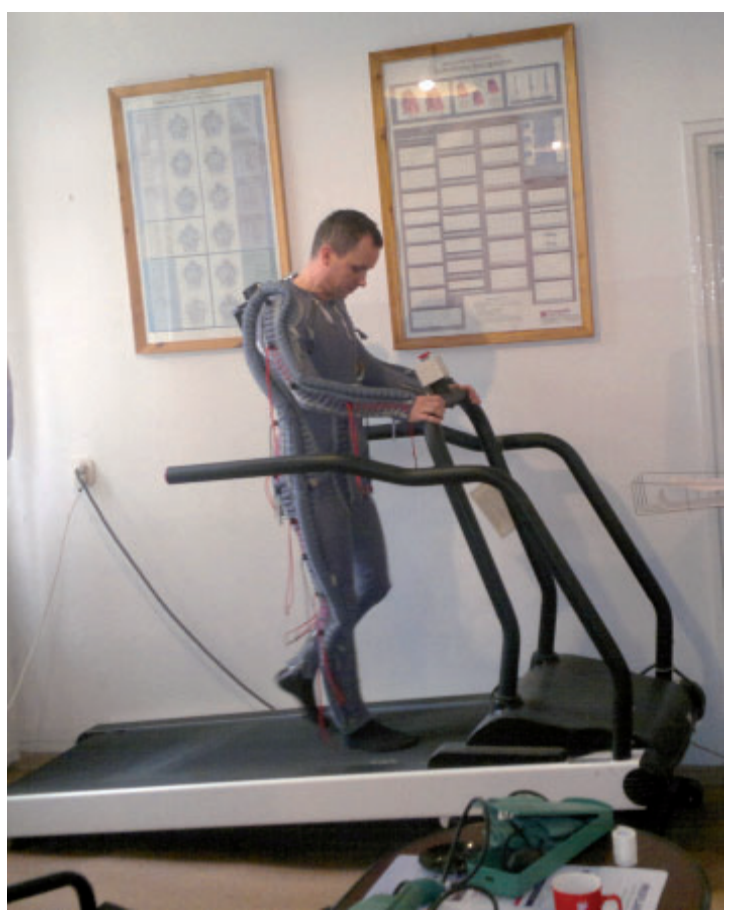

Fig. 3. First subject during experiment (photo presented with participant consent).

statement and was informed about possibility to cease his or her participations anytime. Volunteer subjects were not paid for participation in presented research.

Research was conducted in two main parts: firstly we performed tests on one healthy individual. Subject was male professional soldier 38 years of age, $170 \mathrm{~cm}$ tall, weight $64 \mathrm{~kg}$. He possessed valid fit for military duty assessment from military medical board. Subject during test is shown on fig. 3 .
In second stage results were confirmed with group of 17 healthy volunteers who performed one real kinesitherapy session with use of the HAP suit. Mean age of control group was 29,5 years of age (SD $\pm 4,69$ y; age span was from 23 to 41 y.o.). Eight females and nine males constituted our control group.

Each participant has systolic and diastolic blood pressure measured (with OMRON ${ }^{\mathrm{TM}}$ R7 blood pressure monitor), arterial blood saturation $\mathrm{SpO} 2$ (with finger pulse oximeter). Results were recorded and analyzed with paired t-tests. Measurements of blood pressure were taken 5 min before donning of HAP suit and after that RR and $\mathrm{SpO} 2$ measurements were taken in 3 minutes intervals. Each examination were 24 minutes long. Last measurements were taken 5 minutes after HAP removal.

\section{RESULTS}

As a starter we tried investigation with one volunteer using inflated WUK on the treadmill normally used for stress test cardiography. Physical load was in accordance with Bruce protocol [5]. Physical workload was increased in 3 min intervals. Increase was both in terms of speed and angle of elevation. Stages of exercise test are presented on table (tab. 1) below:

Our subject has attained Stage 4 according to this protocol. Measurements of systolic and diastolic pressures are provided on graph (fig. 4) below:

Control group of 17 volunteer participants were tested in similar manner, but we broadened measured parameters with pulse oximetry. During 24 
Abakumow M. et al. - Safety of use of hight...

Tab. 1. Bruce protocol stages (from [3]).

\begin{tabular}{|c|c|c|c|c|}
\hline Stage & Time (min) & Speed (km/hr) & Gradient (\%) & Metabolic equivalents of tasks \\
\hline 1 & 3 & 2.7 & 10 & 5 \\
\hline 2 & 6 & 4.0 & 12 & 7 \\
\hline 4 & 12 & 6.7 & 16 & 13 \\
\hline 5 & 15 & 8.0 & 18 & 15 \\
\hline 7 & 21 & 9.6 & 22 & 20 \\
\hline
\end{tabular}

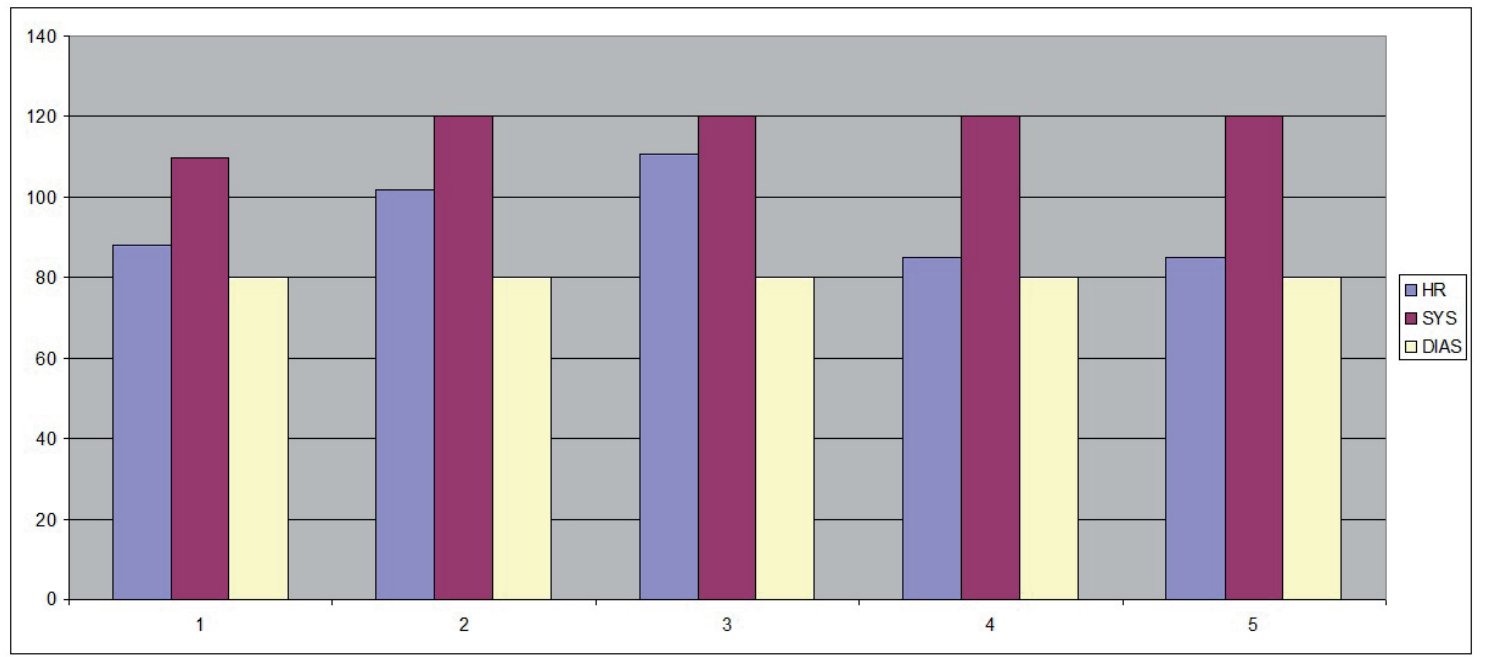

Fig. 4. HR, SYStolic and DIAstolic blond pressure in first subject. 1-baseline before test, 2- results after stage 1, 3- results after stage 2,4- results after stage 3,5 - results after stage 4.
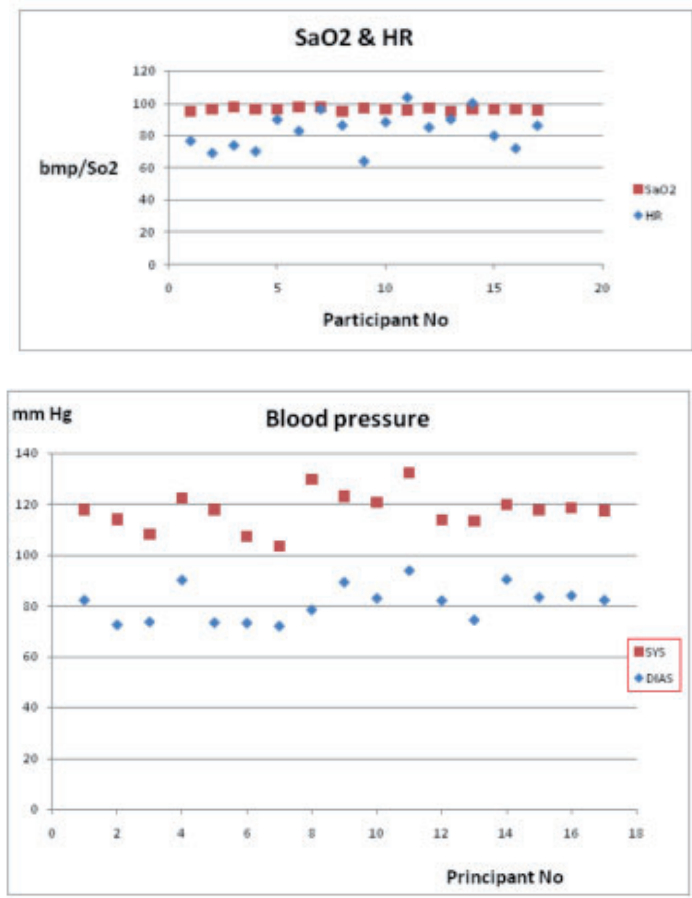

Fig. 5 \& 6. Results of measurements in control group. Means of measurements before and after each session are provided. minutes of measurements period participants performed sets of physical exercises designated for patients (research) group. Results of control group parameters after exercises are presented below (fig. 5 \& 6):

Results obtained indicate, that blood pressure changes (both diastolic an systolic) were on minuscule level (- well below 1\%) (table 2), $p=N S$.

\section{DISCUSSION}

Main safety concern associated with use of compressing devices, especially if they cover chest region is increase of blood pressure resulting from addition of mechanical external pressure to the "normal", heart generated blood pressure. In physiological condition blood pressure is regulated on correct level by reflex from carotid baroreceptors. Distending of carotid artery walls caused by increased blood pressure activates heart inhibition, mainly through vagal nerve. In opposite situation decreased blood pressure on carotid receptor is inhibiting parasympathic activation of vagal nerve thus increasing heart output. This is phenomenon thoroughly studied during head- foot directed acceleration exposures in flight or on the centrifuge. 
Tab. 2. Averaged blood pressures, HR and saturation for 17 persons control group before and after the session.

\begin{tabular}{lcccc}
\hline & Systolic & Diastolic & SaO2 & HR \\
\hline BEFORE & 127,2 & 84,6 & 96,5 & 78,2 \\
\hline AFTER & 130,1 & 82,6 & 96,3 & 84,8 \\
\hline CHANGE (\%) & $\mathbf{0 , 0 2 3}$ & $\mathbf{- 0 , 0 2 4}$ & $\mathbf{- 0 , 0 0 2 1}$ & $\mathbf{0 , 0 8 5}$ \\
\hline
\end{tabular}

This is quite powerful reflex, allowing human body to compensate lower body blood retention up to around four times Earth's gravity. In experiments described in this paper we wanted to check if potential of this reflex going the opposite way i.e. decreasing heart's output in conditions of increased pressure is enough to allow overall blood flow close to normal. Because of systemic nature of the reflex we have chosen wrist blood measurement instead of neck pressure measurements, which are more technically complicated and hardware involved is precluding free movements necessary to perform therapeutic exercises. From centrifuge experiments we know, that use of height compensated limb pressure measurement is sufficient to assess both heart and brain level blood pressure.

All performed measurements have confirmed that use of WUK-90 high altitude protections suit is safe in terms of cardiovascular system parameters in area of pressures used in experiments. We did not encountered any increase of blood pressure which may be considered as a harmful, for participants.
Results obtained suggest that increasing physical pressure on the body is successfully corrected by body homeostasis system. Cardiovascular reflexes from carotid baroreceptors are sufficient to tame increase of hydraulic blood pressure induced by over-the-chest pressure. No breathing difficulties reported by our participants is another prognostic which gives us a rise to conclusion that therapeutic use of high altitude protection suit for kinesitherapy is safe alternative to "normal" sets of exercises planned to perform in therapy of patients.

\section{ACKNOWLEDGEMENTS}

Author wish to thank professor Ewelina Zawadzka-Bartczak and dr Andrzej Orzeł from Military Institute of Aviation Medicine for their invaluable help in performing stage one of described experiment.

\section{AUTHORS' DECLARATION:}

Study Design: Maciej Abakumow, Krzysztof Kowalczuk. Data Collection: Maciej Abakumow. Statistical Analysis: Maciej Abakumow, Krzysztof Kowalczuk. Manuscript preparation: Krzysztof Kowalczuk, Maciej Abakumow. The Authors declare that there is no conflict of interest.

\section{REFERENCES}

1. Hopman MT, Oeseburg B, Binkhorst RA. The effect of an anti-G suit on cardiovascular responses to exercise in persons with paraplegia. Med. Sci. Sports Exerc. 1992; 24(9):984-90.

2. Kogan OG, Naydin V. Medical rehabilitation in neurology and neurosurgery. Medicina. 1988.

3. Lim YC, Teo SG, Poh KK. ST-segment Changes With Exercise Stress. Singapore Med J. 2016; 57(7): $347-353$ doi: 10.11622/ smedj.2016116.

4. Mauritz K-H. General rehabilitation. Current Opinion Neurol Neurosurgeri. 1990; 3:714-718.

5. Miller TD, Askew JW, Anavekar NS. Noninvasive Stress Testing for Coronary Artery Disease. Cardiol Clin. 2014; 32(3):387-404. doi: 10.1016/j.ccl.2014.04.008.

6. Seaworth JF, Jennings TJ, Howell LL, Frazier JW, Goodyear CD, Grassman ED. Hemodynamic effects of anti-G suit inflation in a 1-G environment. J Appl Physiol. 1985; 59(4):1145-51.

7. Voss DE, Ionta MK, Meyers BJ. Proproceptive Neuromuscular Facilitation. 3rd edn. Harper \& Row. New York, 1985.

Cite this article as: Abakumow M, Kowalczuk K. Safety Of Use Of High Altitude Protection Suits For Kinesitherapy - Preliminary Report. Pol J Aviat Med Bioeng Psychol 2019; 25(1): 50-54. DOI: 10.13174/pjambp. 07.12.2020.05 